\title{
Experiencia de aprendizaje-servicio envejecimiento saludable y medio ambiente
}

\section{Verónica Sierra}

Ma. de la Luz Martínez-Maldonado

Mitzy Flores

Sandra Álvarez

\section{Alejandra Montes}

Universidad Nacional Autónoma de México, México

\section{Resumen}

Se trata de una experiencia de aprendizaje-servicio solidario con un grupo de personas mayores residentes de Tlaxcala, México. El objetivo fue promover que las personas mayores desarrollen o fortalezcan su conciencia ecológica y que esta acción impactase positivamente en sus hábitos de reciclaje y envejecimiento saludable. Se efectuó un diagnóstico, planeación, intervención, evaluación y seguimiento de la propuesta educativa. Como resultado, los participantes, además de mejorar sus prácticas de reciclaje, aumentar su conciencia ecológica y adoptar un estilo de vida saludable y sostenible, desarrollaron procesos de organización y participación activa.

\section{Palabras clave}

Aprendizaje-servicio solidario, cultura ambiental, conciencia ecológica, reciclaje, envejecimiento saludable. 


\title{
Solidarity service-learning experience: healthy aging and the environment
}

\author{
Abstract \\ This paper describes a solidarity service-learning experience involving a group of \\ elderly people who live in Tlaxcala. The experience was aimed at promoting the \\ development or reinforcement of the elders' ecological awareness and thus having a \\ positive impact on their recycling habits and promoting healthy aging This educational \\ proposal involved a diagnostic analysis, strategic planning, intervention, evaluation \\ and project monitoring. As a result of the project, the participants improved their \\ recycling habits, increased their ecological awareness, adopted a healthy and \\ sustainable lifestyle, and they were also involved in organizational and active \\ participation processes.
}

\section{Keywords}

Solidarity service-learning, environmental culture, ecological awareness, recycling and healthy aging. 


\section{Introducción}

En el presente trabajo se encontrará una experiencia de aprendizaje-servicio que se llevó a cabo en la asignatura Evaluación y Seguimiento de Proyectos Comunitarios de la licenciatura en Desarrollo Comunitario para el Envejecimiento. La actividad se efectuó en las instalaciones de la Unidad de Atención a la Mujer (UAIM) de la Universidad Autónoma de Tlaxcala. Anticipadamente se realizó un diagnóstico comunitario, se identificó la preocupación y ocupación por la procuración del cuidado del medio ambiente.

Se llevó a efecto la planeación, intervención y evaluación del taller 'Cultura ambiental: una mirada desde el reciclaje'. La propuesta educativa surgió como una estrategia metodológica que permite vincular el aprendizaje curricular con el servicio solidario a la comunidad e impulsa la participación activa de los integrantes.

Esta experiencia fue motivada por la participación y compromiso, por cambiar la realidad de un contexto específico. La propuesta se enfocó en atender un problema global, desde una perspectiva comunitaria. Se reflexionó sobre la poca información en el tema medioambiental y se consideró la causa del deterioro, desgaste y contaminación de nuestro planeta, por ello, es necesario informarse, crear conciencia de la urgente necesidad de cuidarlo y crear acciones para preservarlo (Gracia, y Castillo, 2013).

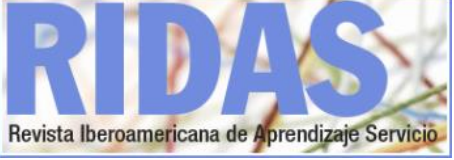

El taller se enfocó en la modificación del nivel de consumo de artículos que producen basura (reducir), dar una segunda oportunidad a aquellos objetos que ya no cumplen su función inicial (reutilizar) y evitar el descontrolado uso de recursos (reciclar).

Dicho lo anterior, el objetivo general del taller fue promover que las personas mayores desarrollen o fortalezcan su conciencia ecológica; y que esta acción impacte positivamente en sus hábitos de reciclaje y envejecimiento saludable.

Los contenidos teóricos que se abordan son aprendizaje-servicio solidario y cómo se aplicó la metodología. Posteriormente, se desarrollarán conceptos como envejecimiento saludable, cultura ambiental, conciencia ecológica y reciclaje. Después se presentan los resultados obtenidos y las conclusiones.

\section{Marco teórico}

El aprendizaje-servicio solidario es una metodología y filosofía que permite que los estudiantes tengan herramientas para la vida. Se trata de aplicar los conocimientos aprendidos en clases, a favor de la comunidad, además, el aprendizaje-servicio solidario, conecta a las instituciones con la comunidad para el mismo beneficio.

Por consiguiente, el aprendizajeservicio solidario es definido como "una forma de aprendizaje por proyectos [...] una estrategia didáctica cuyo objetivo es organizar los contenidos curriculares bajo un enfoque globalizador y significativo que permite relacionar los conocimientos escolares con los de la 
vida cotidiana" (Tapia, 2016, p.47).

El servicio toma fuerza cuando se equilibran aspectos como el servicio a la comunidad, el aprendizaje de contenidos académicos, la práctica de valores y distintos niveles de reflexión y reconocimiento (Puig, 2015).

Entonces, los destinatarios son considerados co-protagonistas, quienes se verán beneficiados de los servicios que ofrecerán los estudiantes, forjarán un vínculo recíproco de enriquecimiento mutuo (Del Campo et al., 2017).

Las intenciones solidarias, incluso el voluntariado, podría confundirse con el aprendizaje-servicio solidario. De acuerdo con Berger Kaye (como se citó en Centro Latinoamericano de Aprendizaje y Servicio Solidario, 2021) en el aprendizaje-servicio solidario los estudiantes participan activamente al comprender, integrar y aplicar conocimientos mientras trabajan para mejorar sus comunidades.

Mientras tanto, Tapia y Ojea (2018) y Del Campo et al. (2017) consideran que el aprendizaje-servicio, está conformado por cinco fases y estas se ven sumergidas, durante todo el proceso, en la reflexión, registro, sistematización, comunicación y evaluación.

La motivación es la parte inicial del proyecto, puede surgir por la demanda de la comunidad, ser considerada como un hecho fortuito o como un proyecto pedagógico. Es necesario determinar quién coordinará la propuesta educativa; presentarse con la comunidad y decidir la forma de trabajo. Si este proceso llegase a darse entre instituciones, la experiencia podría tener un impacto aún mayor, en la comunidad (Del campo et al., 2017).

Durante el diagnóstico, se delimita un problema específico y se proponen soluciones, se reconocen las necesidades, potencialidades, recursos y posibilidades de los participantes. Puede utilizarse cualquier modalidad que se adecúe al contexto de la comunidad, pero el diagnóstico participativo permitirá escuchar la voz de los actores sociales (Del Campo et al., 2017; Tapia y Ojea, 2018).

Diseño y planificación del proyecto es la tercera etapa, en este momento se presenta la propuesta educativa. Es importante construir indicadores de evaluación del proyecto, la planificación debe considerar la naturaleza del proyecto, fundamentación, objetivos de servicio y aprendizaje, responsables del proyecto, destinatarios, beneficiarios, actividades, métodos, técnicas, estimación de tiempos, ubicación, viabilidad, recursos, presupuestos posibles y localización (Del Campo et al., 2017).

En la ejecución, la planeación es puesta a prueba para hacer los ajustes necesarios a la intervención conforme se avanza en el proyecto, asimismo, se debe dar seguimiento a los aprendizajes curriculares y del servicio solidario (Tapia y Ojea, 2018).

El cierre hace referencia a la conclusión de la intervención, entrega de resultados y logros; es el momento para difundir el alcance del proyecto. Se evalúa el aprendizaje curricular y el 
proyecto. Los aspectos básicos para evaluar son la calidad y el impacto del aprendizaje-servicio solidario (Del campo et al., 2017).

El aprendizaje-servicio solidario permite abordar cualquier temática que resulte importante y cubra las necesidades de la comunidad, porque es una metodología flexible ante situaciones no previstas y el contexto de la comunidad. En esta experiencia trabajamos conceptos como envejecimiento saludable, cultura ambiental, conciencia ecológica y reciclaje, los cuales son considerados en la temática medio ambiente, tema objeto de esta experiencia.

Hoy en día, nuestra huella ecológica global supera en un $150 \%$ la capacidad total de la tierra para proporcionar recursos renovables y no renovables a la humanidad. De seguir así, en 35 años con una población que podría llegar a los 9.600 millones de personas en 2050, necesitaremos casi tres planetas para mantener nuestras formas de vida (Ministerio de Medio Ambiente, y Medio Rural y Marino, 2007).

De acuerdo con el Centro Internacional Sobre el Envejecimiento (CENIE, 2018) las personas envejecidas son más vulnerables a los cambios que sufre nuestro medio ambiente, de manera física, social, psicológica y geográfica, por ello, se demanda la comprensión del envejecimiento global en conjunto al deterioro del medio ambiente, así se podrán realizar acciones para minimizar los daños posibles a las diferentes dimensiones del envejecimiento.
Por otro lado, se encuentra el envejecimiento saludable, que se define como:

"el proceso de desarrollo y mantenimiento de la capacidad funcional que permite el bienestar en la vejez [...] tener la posibilidad de vivir en entornos que apoyan y mantienen la capacidad intrínseca y la capacidad funcional es primordial para el envejecimiento saludable" (Organización Mundial de la Salud, 2019, p.1).

Sin duda alguna, la promoción de un envejecimiento saludable es importante para mejorar la condición de vida de muchas personas mayores; el medio ambiente es uno de los implicados en la calidad de vida y su cuidado expresa la oportunidad de mantener la capacidad funcional e intrínseca de las personas al mejorar su entorno.

En efecto, los problemas ambientales surgen por diversos factores, nuestro modelo de vida conlleva gastos de recursos naturales, que con el paso del tiempo aumenta y es insostenible. Algunos efectos de la crisis ecológica ya son visibles, generan un agotamiento de recursos. El consumo de estos es constante y somete a tensión al medio ambiente, lo cual se ve afectado en el ecosistema, flora, fauna, mares, tierra, etc. Esto provoca que las personas no puedan vivir en un ambiente sano y ecológicamente equilibrado (Acurio, 2015).

Por ello, es importante que existan propuestas educativas relacionadas con el medio ambiente, reciclaje, educación 
ambiental, conciencia ecológica y cultura ambiental. Hecho que tiene como base lo expuesto en el debate de la Organización de las Naciones Unidas para la Educación, la Ciencia y la Cultura (UNESCO, 1997) en torno a impulsar una educación para el desarrollo sustentable, relacionándolo con la educación ambiental.

De acuerdo con Severiche-Sierra et al. (2016) la educación ambiental es una herramienta que auxilia a las personas en la toma de conciencia por preservar el medio ambiente, a partir del cambio de valores, conductas y estilo de vida.

Es importante considerar a la cultura como la base de la educación ambiental, esta no es estática y puede definirse y presentarse de diferentes formas, lo cual deja claro que cuando se hable de cultura se mantenga una perspectiva multicultural (SevericheSierra, et al., 2016).

Entonces, entenderemos por cultura a la construcción dinámica y colectiva de un universo y la representaciónentendimiento de éste; considera la reproducción de ideales e identidades, que caracterizan a cada cultura, influenciados por la educación formal e informal (Mata, 2004).

Es así como la cultura ambiental es definida como una transformación de las acciones, creencias y valores orientados a la conservación del medio ambiente, para mejorar la calidad de vida, la propagación de acciones amigables con la naturaleza y la sociedad (Pérez de Villa et al., 2017).

En consecuente, las actitudes y comportamientos de los seres humanos determinarán las proyecciones medio ambientales. De acuerdo con la Organización de las Naciones Unidas para la Agricultura y la Alimentación (FAO) es importante hablar sobre la conciencia ecológica, que aparece cuando los seres humanos, se preocupan por los problemas ecológicos y se informan sobre el tema; la reflexión permitirá el cambio de actitudes y comportamientos con el medio ambiente (Organización de las Naciones Unidas para la Agricultura y la Alimentación, 2009).

Dentro de las acciones más prácticas de manera individual se encuentra el reciclaje, que consiste en la clasificación de basura en función de sus características, acompañado del acceso a la información para el consumo responsable y la oportunidad de utilizar artículos para un segundo fin al que fueron diseñados (Lara, 2008).

Sin duda alguna, mejorar nuestros hábitos de reciclaje mejorará nuestra calidad de vida, permitirá el ahorro de materiales, reducirá los problemas ambientales y brindará la oportunidad de establecer un vínculo sostenible a medida que envejecemos.

\section{Metodología}

Se llevó a cabo un proyecto de aprendizaje-servicio, para el cual se realizó un diagnóstico participativo, planeación, intervención, evaluación y seguimiento de la propuesta educativa. Las técnicas utilizadas para la recolección de datos fueron observación participante, análisis de contenido e investigación documental. 
Dentro del componente de aprendizaje, se conformó un grupo de cuatro estudiantes y dos profesores en la asignatura Evaluación y Seguimiento de Proyectos Comunitarios, de la licenciatura Desarrollo Comunitario para el Envejecimiento, que se imparte en la Facultad de Estudios Superiores Zaragoza, campus III/Tlaxcala, perteneciente a la Universidad Nacional Autónoma de México (UNAM).

El servicio 'Cultura ambiental: una mirada desde el reciclaje' se formalizó en la Unidad de Atención Integral de la Mujer ubicada en el Centro de Tlaxcala Biología de la Conducta (CTBC) de la Universidad Autónoma de Tlaxcala.

La experiencia duró 34 horas en 10 sesiones, del 22 de enero al 11 de marzo del 2020; los miércoles de 9:00 am a 12:30 pm. La última sesión se cumplió de manera virtual el 24 de junio del 2020, respetando el confinamiento por la Jornada Nacional de Sana Distancia, en México. Formaron parte 20 personas, 16 mujeres y cuatro hombres de 41 a 75 años. El 85\% de los participantes tiene estudios profesionales.

El proceso educativo ostentó un componente de investigación, se aplicaron los siguientes cuestionarios para evaluar el impacto: Prácticas de reciclaje, Estado de salud, Cultura ambiental, Escala de depresión geriátrica de Yesavage, Escala de redes de apoyo social para adultos mayores y Escala de generatividad de Loyola.

\subsection{Objetivos de aprendizaje}

Evaluar el proyecto comunitario, de manera ética y responsable, para determinar el impacto de la intervención para establecer estrategias que propicien la mejoría en la calidad de vida de las personas que envejecen y la transformación social.

\subsection{Objetivos específicos}

- Establecer las modalidades de evaluación para una comunidad específica.

- Realizar la evaluación de las actividades desarrolladas en la intervención.

- Discutir las ventajas de la evaluación de cuarta generación.

- Integrar la evaluación al contexto social comunitario.

- Revisar la utilidad de la evaluación en su programa.

- Aplicar el seguimiento de la intervención comunitaria.

\subsection{Objetivo General de la Intervención}

Promover que las personas desarrollen o fortalezcan su cultura ambiental para que esta impacte positivamente en sus hábitos de reciclaje y en un envejecimiento saludable.

\subsection{Objetivos específicos}

- Introducir a las personas envejecidas al tema de cultura ambiental.

- Sensibilizar a las personas envejecidas sobre el consumo responsable de artículos de 
tereftalato de polietileno (PET), así como papel, latas, envases, vidrios, etc.

- Conocer los tipos de reciclaje para llevarlo a cabo.

- Visibilizar los beneficios de llevar a cabo prácticas de reciclaje en el ámbito personal.

\section{Resultados}

Para fines prácticos, los resultados se presentan de manera esquematizada. En el componente de aprendizaje se encuentran las bases generales de la construcción del servicio y aportes curriculares; por otro lado, el componente de servicio contiene logros, productos, aportes y testimonios.

\subsection{Componente de aprendizaje}

Las estudiantes retomaron el diagnóstico realizado previamente y con apoyo de sus profesores, se contactaron con las autoridades de la Unidad de Atención Integral a la Mujer en la UATx, en donde se determinaron las bases del servicio.

Asimismo, se escribió una matriz de marco lógico, una ficha técnica, ocho planeaciones didácticas, la elección del modelo de evaluación y un plan de seguimiento. Al finalizar las sesiones, las alumnas registraban lo sucedido en diarios de campo, lista de asistencias, albúmenes fotográficos $y$

autoevaluaciones.

El primer momento de aplicación de instrumentos, se realizó al inicio del taller, con la finalidad de evaluar los conocimientos previos de los participantes. Se llevó a cabo el día 22 de enero del 2020 con un total de 20 participantes, de los cuales 18 sabían leer y escribir, por ello fue una auto aplicación. A los dos participantes que tenían problemas para leer los instrumentos, se les aplicó de manera verbal.

Si bien no se logró realizar una valoración final; las autoevaluaciones, diarios de campos y multimedios, permitieron conocer el impacto del servicio, dando como resultado la adopción de un envejecimiento saludable, un incremento de prácticas de reciclaje, preocupación por el medio ambiente y cambio de actitudes ambientales.

Por otro lado, las estudiantes practicaron su capacidad para adaptar los contenidos del taller en un nuevo escenario, al inicio se planearon actividades de contribución a la comunidad, sin embargo, los participantes manifestaron que preferían construir lombricompostas para reducir sus desechos orgánicos, utilizarlos para plantar alimentos 0 plantas ornamentales en casa generando un beneficio propio y comunitario al reducir los desechos, después invitar a vecinos y familiares a formar sus propias compostas o escalar la práctica colectiva.

El mayor obstáculo que enfrentó este proyecto fue la llegada de la pandemia por COVID-19, ya que se tuvo que cancelar la última sesión del taller y la evaluación pos-test.

Como recomendaciones para futuras 
intervenciones, se concluyó hacer una lectura objetiva del impacto del servicio por medio de una evaluación final con instrumentos estandarizados. También, se propone difundir la importancia de la autoevaluación para complementar la información y mejorar el análisis de datos en casos extraordinarios como el actual.

\subsubsection{Aportes curriculares}

A manera de aporte y aprendizaje curricular, las estudiantes expusieron el servicio a medios de comunicación de la región, se difundió la experiencia y se publicó en el periódico El Sol de Tlaxcala y fue transmitido en la estación de radio de la UATX.

\subsection{Componente de servicio solidario}

\subsubsection{Logros}

a) Conformación del grupo Tonatiuh, anteriormente practicaban juntos Taichi y recibían atención médica juntos, sin embargo, no se propiciaba un espacio de reflexión y diálogo.

b) Decidir el nombre y lema del grupo, reforzó la cohesión grupal e incentivo el sentido de pertenencia. La reflexión grupal sobre el lema motivó a los participantes a informarse sobre la creación de una asociación civil.

c) Surgieron procesos de organización y participación activa, al realizar actividades a fines.

\section{Rilis.}

El primer momento fue la gestión, por parte de los miembros del grupo, a una visita guiada a Morphoplast (empresa dedicada al reciclaje de botellas) y solicitaron la ubicación de tres módulos de recolección en diferentes municipios de Tlaxcala.

El segundo momento fue la gestión de una ponencia sobre procesos hídricos y captación de agua en casa. Esta ponencia, impartida por un especialista, permitió que el grupo ampliara sus redes de apoyo social.

El tercer momento de organización (no pudo ser llevada a cabo por la contingencia sanitaria para contener el contagio de coronavirus) fue la reservación de una visita guiada a un centro vacacional, en donde se ofrecen talleres sobre alimentación sostenible por medio huertos en casa.

d) Otro logro fue comenzar las bases de organización, entre los participantes, para conformar una asociación civil orientada al cuidado del medio ambiente y al envejecimiento saludable.

\subsubsection{Productos}

Los participantes crearon y diseñaron productos como un generador de energía para electrodomésticos impulsado por agua, juguetes, organizadores, obras de arte, decoración del hogar, filtros de agua caseros, captadores de agua de lluvia y 
reciclaje de basura inorgánica.

Con respecto al reciclaje orgánico, los productos obtenidos fueron artículos de higiene personal (pasta de dientes, desodorante, champú, jabón corporal y acondicionador) y de limpieza del hogar (insecticida, limpiador multiusos, suavizante de telas y ambientador de habitaciones).

Asimismo, diseñaron y difundieron un directorio sobre los centros de reciclaje cercanos a su comunidad.

Posteriormente, crearon un manual didáctico para reciclar plástico, cartón, papel y vidrio y lo compartieron con sus vecinos y amigos; para finalizar discutieron acerca de la pertinencia del manual para despertar la conciencia ecológica.

Por otra parte, escribieron una lista de actividades que se pueden realizar desde casa para fortalecer la cultura ambiental y brindaron apoyo a compañeros que solicitaron recomendaciones para iniciar una conversación con sus familiares, sobre temas a fines.

En cuanto a los productos de difusión de información realizaron una escena de teatro con el objetivo de visibilizar la importancia de la cultura ambiental y el cuidado del medio ambiente manteniendo un envejecimiento saludable.

Finalmente, produjeron podcasts sobre el cuidado del agua y la importancia de reciclar, adoptando así, nuevas formas de difusión de información en la comunidad.

\subsubsection{Aportes al taller}

Se presentó una ponencia sobre la conciencia ecológica por un especialista del CTBC y se realizó una ponenciapráctica sobre lombricomposta por alumnos de Biología, de la UNAM. Dichos encuentros fueron avalados por la UATx, brindando a los alumnos construir una base curricular, a pesar de no ser beneficiarios directos del aprendizaje-servicio.

\subsubsection{Testimonios}

- "[...]yo realmente, siempre he tenido el ideal de que una persona de una edad avanzada no debe estar postrada en una cama, aunque sea con un bastón caminar, aunque sea para hacer sus necesidades, tener esa libertad, pero ¿cómo se logra? Con una alimentación balanceada, ejercicios, recreación y todo para estar saludable, que mejor que hacerlo tú mismo, plantar tú comida. Aunque sea con bastón o lo que tenga, seguiré" (L. García, comunicación personal, 29 de enero de 2020).

- "Aún faltan políticas públicas que realmente funcionen y regulen a las empresas que contaminan el medio ambiente, pero para dejar de echarle la culpa a las empresas, debemos pensar qué hacer nosotros a nivel comunitario. Sí, el gobierno y la empresa tienen responsabilidad, pero también nosotros. La opción, es reciclar y tener conciencia sobre lo que consumimos. Ya después se verá 
lo demás. El cambio comienza con uno mismo y así podemos ir invitando a más personas a cuidar el medio ambiente y aumentar nuestra conciencia ecológica" (G. Jiménez, comunicación personal, 12 de febrero de 2020).

\section{Conclusión}

Desarrollar la experiencia de aprendizaje-servicio permitió la apropiación de conocimientos curriculares, en el caso presentado, fue sobre evaluación y seguimiento de proyectos comunitarios. Resultó significativa tanto para los beneficiarios como para los estudiantes, se atendió un problema global desde la comunidad. Se pusieron en práctica valores como la solidaridad, esfuerzo, tolerancia, diálogo, responsabilidad, se desarrollaron habilidades y capacidades que permitirán la construcción de competencias para el futuro.

La metodología aprendizaje-servicio fue flexible para atender sugerencias sobre las actividades prácticas del taller y la adaptación al contexto de la comunidad. Sin duda, los inconvenientes no dejaron de presentarse, pero impulsó a las estudiantes y a los participantes a generar procesos de resolución de conflictos y de adaptación al cambio.

Por otra parte, se concluye que el servicio solidario fomentó la organización y la participación de los beneficiarios para adoptar hábitos que permitan el cuidado de medio ambiente, promover un envejecimiento saludable, iniciar el reciclaje de basura en casa, la reutilización y la compra consciente de productos. Asimismo, se potenció la cohesión grupal, la comunicación asertiva y la iniciativa de generar proyectos que promueven la generatividad, el fortalecimiento de las redes de apoyo social y la adopción de un envejecimiento saludable.

\section{Referencias bibliográficas}

Acurio, C. A. (2015). El reciclaje de los desechos sólidos y su incidencia en los derechos de las personas para el buen vivir (Tesis de grado). Universidad Técnica Estatal de Quevedo, Quevedo, Ecuador. Recuperado de https://repositorio.uteq.edu.ec/bitstrea m/43000/720/1/T-UTEQ-0041.pdf

Centro Internacional Sobre el

Envejecimiento (CENIE). (27

Septiembre de 2018). El medio ambiente también tiene algo que decir. Recuperado de https://cenie.eu/es/blog/el-medioambiente-tambien-tiene-algo-que-decir

Centro Latinoamericano de Aprendizaje y Servicio Solidario. (20 de enero de 2021). ¿Qué es "aprendizaje-servicio"? Aprender sirve, servir enseña.

Recuperado de https://clayss.org.ar/aprendizajeservici o definiciones.html

Del campo, G., Gimelli, A. y Tapia, M. N. (2017). Escuelas para el encuentro.

Cómo desarrollar proyectos de aprendizaje-servicio solidario. Ciudad Autónoma de Buenos Aires, Argentina: CLAYSS. Recuperado de:

http://www.clayss.org.ar/04 publicacio nes/Manual CLAYSS Scholas.pdf 
Gracia, J. y Castillo, R. (2013).

Educación Ambiental y Personas

Mayores. Guías Didácticas de Educación

Ambiental. Junta de Andalucía.

Recuperado de

http://www.juntadeandalucia.es/medio ambiente/portal web/web/temas ambi entales/educacion ambiental y formac ion nuevo/mayores/geam personas $\mathrm{m}$ ayores.pdf

Lara, J. (2008). Reducir, Reutilizar y Reciclar. Elementos: ciencia y cultura, 15(69), 45-48. Recuperado de https://www.redalyc.org/pdf/294/2940 6907.pdf

Mata, A. (2004). Transformación de la cultura ambiental mediante la docencia universitaria. Biocenosis, 18(1-2), 129134. Recuperado de https://revistas.uned.ac.cr/index.php/bi ocenosis/article/view/1399/1468

Ministerio de Medio Ambiente, y Medio Rural y Marino. (2007). Análisis de la huella ecológica en España. Recuperado de

https://www.footprintnetwork.org/cont ent/images/uploads/Huella\%20ecologic a\%20de\%20Espana.pdf

Organización de las Naciones Unidas para la Agricultura y la Alimentación. (FAO). Glosario de Agricultura Orgánica. Recuperado de http://www.fao.org/tempref/docrep/fao 1012/k4987t/k4987t.pdf

Organización de las Naciones Unidas para la Educación, la Ciencia y la Cultura (UNESCO). (1997). Educación para un futuro sostenible: una visión transdisciplinaria para una acción concertada. Documento de la
Conferencia International Conference on Environment and Society: Education and Public Awareness for Sustainability. Thessalonika, Grecia. Recuperado de https://unesdoc.unesco.org/ark:/48223 Lpf0000110686 spa

Organización Mundial de la Salud. (2019). Década del Envejecimiento Saludable 2020-2030. Primer informe de progreso, marzo del 2019.

Recuperado de

https://www.who.int/docs/defaultsource/documents/decade-of-healthageing/decade-healthy-ageingupdate1-es.pdf?sfvrsn $=d 9 c 407330$

Pérez de Villa, Y., Bravo, N. y Valdés, I. (2017). La cultura ambiental en los procesos universitarios. Universidad y Sociedad, 9(5), 154-164. Recuperado de

https://rus.ucf.edu.cu/index.php/rus/ar ticle/view/726/826

Puig, J. M. (Coord.). (2016). ¿Cómo realizar un proyecto de aprendizaje servicio? 11 ideas clave. Barcelona, España: Graó

Severiche-Sierra, C., Gómez-

Bustamante, E. y Jaimes-Morales, J. (2016). La educación ambiental como base cultural y estrategia para el desarrollo sostenible. Telos, 18(2), 266281. Recuperado de https://www.redalyc.org/pdf/993/9934 5727007.pdf

Tapia, M.N. (Ed.). (2016). Cómo desarrollar proyectos de aprendizaje $y$ servicio solidario en educación media: secundaria y enseñanza técnica. Ciudad Autónoma de Buenos Aires, Argentina: CLAYSS. Recuperado de 
https://www.clayss.org.ar/uruguay/2 S

ecundario.pdf

Tapia, M.N. y Ojea, B. (2018). Guía

para desarrollar proyectos de

aprendizaje-servicio solidario : edición

Perú. Ciudad Autónoma de Buenos

Aires, Argentina: CLAYSS. Recuperado

de

https://www.clayss.org.ar/04 publicaci

ones/Manual Peru para web.pdf 